\title{
Perceptions of a crisis service by referrers and clients
}

\author{
Colin Murray Parkes, Senior Lecturer in Psychiatry, The London Hospital Medical \\ College, Turner Street, London E1 2AD
}

There are many kinds of crisis service. Most are open only to people who have been diagnosed as mentally ill. They facilitate rapid admission to short-stay inpatient services with the back-up of multidisciplinary teams working in the community. Others attempt to keep patients out of hospital by providing support to patients and their families in the community. Several have demonstrated striking reductions in the rate and duration of hospital admissions without detriment to the subsequent health or social adjustment of the patient.

The Tower Hamlets Crisis Intervention Service (CIS) was set-up as a joint health and social services project in 1976. A multidisciplinary team visits families in crisis to assess their needs and, when appropriate, provides short-term support in the community. It differs from most other crisis services in attempting to prevent as well as treat mental ill health. It accepts referrals from a range of professional care givers and is not limited to people with overt mental illness, defining a crisis as any situation which creates distress in a family. Although the team can make use of the full range of psychiatric and social work services, the preferred method of treatment is short-term family therapy aimed at reinforcing or restoring the family as a support system to its members. Most assessment and treatment is carried out in the home. Case-notes are 'family' and 'problem' oriented.

This paper reports the results of an evaluation of the service as seen through the eyes of referrers and clients of the service.

\section{The study}

Information for this evaluation was obtained in three ways:

(a) Systematic analysis of the case-notes of 118 clients referred for the first time to the CIS (94 women and 24 men) between January 1984 and May 1985.

(b) Interviews with 29 GPs, 30 social workers (SWs) and 23 other professionals (82 in all) who had made referrals to the service during that period.

(c) Interviews in their homes with 107 clients of the service referred for the first time to the service during the period of study.

\section{Findings}

Of clients, $80 \%$ were women with $79 \%$ under age 40 (mean age: women 32, men 33). A half had partners (40\% married, $12 \%$ cohabiting) with $29 \%$ single, $15 \%$ separated or divorced and $4 \%$ widowed.

Reasons for referral given by the referrers included "threatened separation of family member" $50 \%$; "suspected or actual psychosis" $39 \%$; "threatened or attempted suicide" $28 \% ; 87 \%$ gave "depression or distress in the family", and $18 \%$ other reasons. Three-quarters were referred because of risk of suicide, violence or psychosis.

Although a psychiatrist subsequently made a psychiatric diagnosis in $79 \%$ of clients, psychoses were diagnosed in only $13 \%$. Other diagnoses were personality disorder $(21.5 \%)$; neurotic depression $(14 \%)$; anxiety state $(9.7 \%)$; brief depressive reactions $(8.4 \%)$; alcohol dependence or abuse $(7.5 \%)$; and phobic state (7.4\%). Other conditions occurred in less than $5 \%$ of cases.

More useful from the point of view of management was the analysis of problems. Nearly two-thirds of those with partners had major problems in relating to those partners whereas $41 \%$ of those without partners had suffered the long-standing loss and a quarter the recent loss of an adult person to whom they were attached. A third complained of social isolation or alienation from their families and a similar proportion of alcohol related problems.

Problems with children were common and six adolescents all had problems relating to their parents.

\section{Management}

All clients were visited in their homes by a team of professionals (usually two). In $60 \%$ a psychiatrist was a member of the team, $55 \%$ a community psychiatric nurse, and $52 \%$ a social worker. In addition to the client other family members were present at two-thirds of the initial (assessment) meetings (38\% spouses, $33 \%$ children, $16 \%$ parents, and $11 \%$ siblings).

On average, families received 6.8 visits from the team over approximately 12 weeks. In addition $10 \%$ received an average of 4.5 individual psychotherapy sessions. Antidepressants were prescribed for $13 \%$ of clients, major tranquillisers for $6 \%$, and minor 
tranquillisers for $3.5 \%$. Twenty-nine per cent were referred on to other agencies, including $7 \%$ admitted for in-patient psychiatric care for a mean of $\mathbf{4 4}$ days each.

\section{Evaluation}

Effectiveness. Of referrers, $78 \%$ thought that crisis intervention had led to the action they had hoped for; $79 \%$ thought that distress in the family had reduced or ceased; nearly a half thought that the risk of further crises had been reduced and in a quarter the need for psychiatric admission had been reduced or eliminated.

Three-quarters of clients had found the service "helpful or very helpful" and two-thirds, asked "How have things been since the team stopped visiting?," answered "better" or "much better" (17\% said "worse"). Among $\mathbf{4 0}$ clients who were asked "What do you think would have happened if you had not been referred to the Crisis Service?", a quarter said that they might have committed suicide, a quarter that they would have become mentally ill, and $10 \%$ that they would have left home.

Features most often thought to have contributed to the effectiveness of the teams were support to and increased understanding by the family and the opportunity to talk through problems.

Efficiency. The annual cost of the service at the midpoint of the evaluation (1984-5) was $£ 35,000$ or about $£ 300$ per family. This compared with the cost at that time of a hospital bed of about $£ 20,000$ but we have no way of knowing how much time these people would have spent in hospital if the CIS had not been available and the unending pressure on in-patient services in this district meant that any bed that is freed-up from one source is quickly filled from another.

Could similar results have been achieved by sending only one professional? The team argue that at least two professionals are needed to meet the needs of a family and to avoid undue medicalisation. By sending a 'family' (the CIS team) to meet the client's family they attempted to provide a system of care which supports and educates, and to reduce the likelihood that the family will be unable to cope with future crises. In general this expectation is born out by the results. Although half the families reported 'some', and 29\% 'many', further problems, most had been resolved without the need for further professional help. Only $21 \%$ had been re-referred to the CIS between discharge and follow-up 15 months later, requiring an average of only two further interviews, half of them by one member of the team. There has been no tendency for the CIS to 'silt-up' over the years with long-term mentally ill patients who are more appropriately helped by rehabilitation services.
Appropriateness. Ninety-three per cent of GPs, $97 \%$ of SWs and $83 \%$ other professionals asserted that the client's home is the most appropriate venue in which to assess crises in the family. Only three caregivers, two of them consultant psychiatrists, thought 'hospital' the most appropriate place.

Most GPs and SWs thought a psychiatrist the most appropriate person to help families in crisis, yet $40 \%$ of the teams did not include a psychiatrist. Close study of the reports on 46 clients whose team did not include a psychiatrist revealed no misdiagnoses or mistreatments. None of these clients subsequently required admission, day care or out-patient care, and none were referred to the emergency clinic. No clients or referrers criticised the service for not including a psychiatrist although there were a few criticisms that the team, by including a doctor, may sometimes be medicalising normal life crises.

The short-term therapy was approved by half the clients who thought the number of visits "about right", a third would have liked more and $13 \%$ less visits from the team; $91 \%$ agreed with the decision to end therapy and were satisfied with the advice given at that time.

Among 14 psychotic patients referred, six were subsequently admitted for in-patient psychiatric care, one referred to day hospital care and two refused further visits and further action was not deemed appropriate. The remaining five accepted and seemed to benefit from the care of the CIS. It would seem that, while many psychoses require hospital care, some benefit from the family support provided by the CIS team which was usually valued even if it led to admission.

Equitability. The service was criticised on the grounds that it does not accept referral of children, elderly people, or those already receiving psychiatric care (unless referred by their psychiatrist). Staff argue that satisfactory alternative services already exist for these excluded groups. They see the CIS as complementing, not competing with other services.

It was also suggested that the service should be open to clients without a family in Tower Hamlets. This questioned the basic assumption of a familyoriented service and caused the researcher to look closely at the 34 clients whose families declined or were not available to meet with the team. They seem to have benefited from and valued the help of the service as much as those with co-operative families! It may be that those who lack family support are in just as much, if not greater need, of counselling and support but also that individual one-to-one counselling would have been a more cost-effective way of meeting their needs.

Acceptability. The CIS attempts to be acceptable to the local population by visiting the client's home, operating from a base in the community, encouraging 
non-psychiatric referrals and not including a psychiatrist in $\mathbf{4 0 \%}$ of teams. Only one referrer thought that a client had been stigmatised as a consequence of referral and $55 \%$ thought that stigma had been reduced (perhaps because psychiatrists, when present, often reassure families that the client is not mentally ill). Even so, a third of clients had been apprehensive about the first visit and $13 \%$ had found this "difficult" (two-thirds, on the other hand, found it "easy"). Clients from ethnic minority groups may find the service less acceptable than those from the parent population because of the cultural differences that exist between clients and CIS staff (and staff of most other services). One GP who works mainly with patients from Bangladesh had made no referrals to the service on the grounds that he did not think it suitable for his patients and the proportion of clients from Bangladesh referred to the CIS (3.1\%) is substantially smaller than the proportion in the parent population (about $9 \%$ ).

Accessibility. Most referrers rated the CIS as "easily accessible at all times". This was most apparent to social workers and other non-doctors whose direct access to other psychiatric services is limited since most only accept referral from a GP or other doctor.

The CIS is not currently able to provide 24 hour cover and most referrers think this is unsatisfactory. On the other hand, only $5 \%$ of clients were dissatisfied with delays in setting-up the initial visit.

One referrer and several clients suggested that the CIS should be open to self-referral. This would improve accessibility but staff feared that it would lead to overload and to inappropriate use of a service which prefers to back-up the open doors provided by GPs, SWs and the existing emergency clinic.

If primary carers are to continue to control access to the service, it is important for them to have a good knowledge of it. Of referrers, $47 \%$ agreed that there was a lack of publicity and, of the seven GPs who had made no referrals to the service, five requested further information and only one said that he would "probably not" make a referral in the future.

\section{Comment}

The CIS serves a population who are younger and more likely to be female and have partners than most psychiatric in-patients and long-term mentally ill people. It focuses, therefore, on a group who may have special potential for the prevention of mental illness in future generations. On the other hand, the service may not be reaching the older, unattached members of the community whose need for psychiatric help is greatest.

In the absence of a satisfactory control group, any conclusions drawn regarding the efficacy of the service provided by the Tower Hamlets CIS must be cautious.

Overall comments by clients and referrers tended to be very positive and to reflect a high degree of satisfaction with the service. The findings strongly suggest that the service substantially reduces the risk of suicide, mental hospital admission and family break-up, while improving the family's ability to cope with future crises. Working with the service was also seen as a valuable training experience for members of the caring professions.

Criticisms of the service were few. They included a wish for more information to referrers and more clarity in the information given to them about the service. Several clients would have liked the support from the service to have continued for longer and thought, with referrers, that the service should be made available to a wider range of clients and extended to other districts. Doubts were expressed concerning the ability of staff from the UK to understand and communicate with families from Bangladesh.

These findings have persuaded the DHS to continue funding for a consultant in community psychiatry in the Borough. A new psychiatric service for patients from Bangladesh is being set-up with Sylheti-speaking staff and it is hoped that this group will take part as team members within the CIS when appropriate.

\section{Acknowledgements}

This study was carried out with the help of a grant from the Research Department of the Department of Health. Thanks are due to Dr Gillian Waldron for carrying out the diagnostic assessments, to Diana Brendenkamp for sensitive interviewing, and to the clients and referrers kind enough to answer our questions.

A full list of references and further details of the study are available on request to the author. 